\title{
Repeated injection of PEGylated solid lipid nanoparticles induces accelerated blood clearance in mice and beagles
}

This article was published in the following Dove Press journal:

International Journal of Nanomedicine

II June 2012

Number of times this article has been viewed

\section{Yongxue Zhao \\ Long Wang \\ Mina Yan \\ Yanling Ma \\ Guangxi Zang \\ Zhennan She \\ Yihui Deng}

College of Pharmacy, Shenyang Pharmaceutical University, Shenyang, Liaoning, People's Republic of China
Correspondence: Yihui Deng College of Pharmacy, Shenyang Pharmaceutical University, 103 Wenhua Road, Shenyang II0016, Liaoning, People's Republic of China Tel/fax +862423986316

Email pharmdeng@gmail.com
Abstract: Surface modification of nanocarriers with amphiphilic polymer polyethylene glycol (PEG), known as PEGylation, is regarded as a major breakthrough in the application of nanocarriers. However, PEGylated nanocarriers (including liposomes and polymeric nanoparticles) induce what is referred to as the "accelerated blood clearance (ABC) phenomenon" upon repeated injection and consequently they lose their sustained circulation characteristics. Despite this, the present authors are not aware of any reports of accelerated clearance due to repeated injection for PEGylated solid lipid nanoparticles (SLNs), another promising nanocarrier. This study investigated the pharmacokinetics of PEGylated SLNs upon repeated administration in mice; moreover, the impact of circulation time on the induction of the $\mathrm{ABC}$ phenomenon was studied in beagles for the first time. The ABC index, selected as the ratio of the area under the concentration-time curve from time 0 to the last measured concentration of a second injection to that of the first injection, was used to evaluate the extent of this phenomenon. Results showed that the PEGylated SLNs exhibited accelerated clearance from systemic circulation upon repeated injection, both in mice and in beagles, and the ratio for the different time intervals, which showed that the $\mathrm{ABC}$ index exhibited significant difference within 30 minutes following the second injection, was good enough to evaluate the magnitude of $\mathrm{ABC}$. This $\mathrm{ABC}$ index indicated that the $10 \mathrm{~mol} \%$ PEG SLNs with a suitable prolonged circulation time induced the most marked ABC phenomenon in this research. This study demonstrated that, like PEGylated nanocarriers such as liposomes and polymeric nanoparticles, PEGylated SLNs induced the $\mathrm{ABC}$ phenomenon upon repeated injection - the beagle was a valuable experimental animal for this research. Furthermore, the authors considered that a relatively extended circulation time of the initial dose may be the underlying major factor determining the induction of the $\mathrm{ABC}$ phenomenon.

Keywords: SLNs, polyethylene glycol, ABC phenomenon, circulation time

\section{Introduction}

Nanomedicine, the medical application of nanotechnology, holds considerable promise as far as drug delivery systems are concerned. Over the last few decades, there has been dramatic development of nanocarriers in nanomedicine, including liposomes, polymersomes, and polymeric nanomicelles, opening up new possibilities for cancer imaging, molecular diagnosis, and targeted therapy. ${ }^{1}$ Surface modification of nanocarriers with amphiphilic polymer polyethylene glycol (PEG), known as PEGylation, is regarded as a major breakthrough in the application of nanocarriers. ${ }^{2}$ It is widely believed that the steric stabilization provided by PEG polymers confers a relative "invisibility" to the nanocarriers, which is reflected in a reduced recognition 
by the mononuclear phagocyte system and a prolonged blood circulation time; because of this, the PEGylated nanocarriers have attracted increasing attention. ${ }^{3-5}$ However, it has recently been reported that some PEGylated liposomes produce an unexpected phenomenon: PEGylated liposomes including PEGylated cationic liposomes containing nucleic acids have been found to lose their long-circulating characteristics upon repeated administration at certain time intervals in the same animal (referred to as the "accelerated blood clearance [ABC] phenomenon") ${ }^{6-12}$ Furthermore, it is worth noting that this phenomenon was triggered by preadministration with not only PEGylated liposomes but also PEG-containing polymeric nanoparticles ${ }^{8,9}$ and polymeric micelles. ${ }^{10,13}$ These results imply that this unexpected phenomenon, being a potential challenge for PEGylated nanocarrier applications, would greatly compromise the benefits of nanoparticles during cancer diagnosis and drug or gene-mediated therapy.

Solid lipid nanoparticles (SLNs) have been used as a new colloidal carrier since $1991 .{ }^{14}$ Given the low intrinsic toxicity and the biodegradability of the lipids used in SLNs, as well as the increased drug stability and the controlled drug release, it is not surprising that this relatively new class of drug carriers is quickly being adopted for the delivery of a variety of antineoplastic agents. ${ }^{15-18}$ The SLN systems have also been shown to cross the blood-brain barrier ${ }^{15-17}$ and to bypass the resistance mechanism involved in multidrug resistance ${ }^{19-22}$ - therefore, they have unique advantages as drug carriers in cancer therapy. It is expected that modified SLNs with PEG derivatives will be developed and used to take advantage of these properties. However, in view of the ABC phenomenon induced by PEGylated nanocarriers, study of the pharmacokinetics of PEGylated SLNs upon repeated administration is of great importance. For this research, anticancer drugs cannot be used as model drugs - this is because of their toxic effects on the involved immune system. ${ }^{23-26}$ Therefore, tocopheryl nicotinate (TN) was employed as an indicator in the present study by virtue of its lack of effect on the immune organs. Moreover, TN can be incorporated into SLNs with a high payload and stability, which helps to mimic the actual elimination of SLNs.

The ABC phenomenon has already been widely reported in mice, ${ }^{27}$ rats, ${ }^{28,29}$ rabbits, ${ }^{30}$ and rhesus monkeys. ${ }^{7}$ Instead of these animals, the beagle was selected as the primary experimental subject in the present study. This was because of the beagle's relatively high blood volume, meaning collecting many blood samples over a short period time has little effect on physical function, and it is easy to monitor possible reactions throughout the entire experimental process. Both of these points were of great benefit for the ABC investigations undertaken.

The prolonged blood circulation time is the most important characteristic of PEGylated nanocarriers; however, despite extensive studies on the $\mathrm{ABC}$ phenomenon, it is still not clear whether the circulation time affects the induction of $\mathrm{ABC}$. In the current study, the authors first investigated whether the $\mathrm{ABC}$ phenomenon was induced by repeated injection of PEGylated SLNs in mice. Following this, the ratio of $\mathrm{AUC}_{(0-\mathrm{t})}$ (ie, area under the concentration-time curve from time 0 to the last measured concentration) for the second injection to that of the first one was selected as the ABC index, used to evaluate the effects of circulation time on the induction of the $\mathrm{ABC}$ produced in beagles.

\section{Material and methods Material}

Glycerol monostearate (Sigma-Aldrich, St Louis, MO) was used as the matrix for the SLNs. Injectable soybean lecithin (S75) was purchased from Lipoid GmbH (Ludwigshafen, Germany) and N-(Carbonyl-methoxypolyethyl-eneglycol2000)-1,2-distearoyl-sn-glycero-3-phosphoethanolamine ( $\mathrm{mPEG}_{2000}$-DSPE) was purchased from Genzyme Corporation (Cambridge, MA). TN was purchased from Northeast Pharmaceutical Group Co, Ltd (Shenyang, Liaoning, China). Sephadex ${ }^{\circledR}$ G-50 medium was supplied by Sigma-Aldrich. The ethanol was of pharmaceutical grade; all other reagents were of chromatographic grade.

\section{Animals}

Male Kunming mice (each weighing 20-25 g) and male beagles (each weighing 10-12 kg) were purchased from the Laboratory Animal Center of Shenyang Pharmaceutical University (Shenyang, Liaoning, China). All animal care and experiments were carried out in accordance with the guidelines of the Animal Welfare Committee of Shenyang Pharmaceutical University.

\section{Preparation of the PEGylated SLNs}

The melt-emulsification process was used to make the SLNs. Briefly, glycerol monostearate, $\mathrm{S} 75, \mathrm{mPEG}_{2000}-\mathrm{DSPE}$, and TN were mixed and dissolved in ethanol at $65^{\circ} \mathrm{C}$ and the solvent was then removed. The melted mixture was then dispersed in 5\% glucose solution, under mechanical agitation, to achieve a final phospholipid (S75 and $\mathrm{mPEG}_{2000}$-DSPE) concentration of $5 \mu \mathrm{mol} \cdot \mathrm{mL}^{-1}$. The warm primary emulsion was further treated using a laboratory ultrasonic cell pulverizer (JY92-II; Ningbo Scientz 
Biotechnology Co, Ltd, Ningbo, Zhejiang, China), for at least a 2-minute cycle $(200 \mathrm{w})$ and an additional 3-minute cycle $(400 \mathrm{w})$. Following sonication, to ensure homogeneity in size, the suspensions were then passed through polycarbonate membranes with a pore size of $0.22 \mu \mathrm{m}$. The components of each formulation are presented in Table 1.

\section{Determination of encapsulation efficiency}

The untrapped TN was removed by size exclusion chromatography, using a Sephadex G-50 mini-column $(10 \times 70 \mathrm{~mm})$ with double-distilled water as the eluant. Briefly, $100 \mu \mathrm{L}$ of SLN suspension was loaded onto the Sephadex G-50 mini-column and then eluted with $100 \mu \mathrm{L}$ of double-distilled water four times. The concentration of TN in the SLNs was determined by high-performance liquid chromatography (HPLC) after dissolving the SLNs obtained from gel chromatography in methanol. The HPLC conditions were the same as for the method described for the pharmacokinetics studies. Encapsulation efficiency of the SLNs was calculated as the percentage of TN remaining in the SLNs following elution.

\section{Determination of the incorporated ratio of $\mathrm{mPEG}_{2000}$-DSPE in SLNs}

The ultrafiltration method was employed to separate $\mathrm{mPEG}_{2000}$-DSPE not incorporated into SLNs. Free $\mathrm{mPEG}_{2000}{ }^{-}$ DSPE was separated by passing the SLN suspension through the stirred ultrafiltration cells (Merck Millipore, Billerica, MA), and the molecular weight cutoff of the ultrafiltration membrane (Shanghai Yadong Resin Co, Ltd, Shanghai, China) was $100 \mathrm{kDa}$. The amount of $\mathrm{mPEG}_{2000}$ DSPE was determined according to Shimada et al's ${ }^{31}$ procedure, with some modifications. ${ }^{32}$ Briefly, $10 \mathrm{~mL}$ of sodium nitrate-picrate solution (sodium nitrate $3.33 \mathrm{~mol} \cdot \mathrm{L}^{-1}$, picric acid $0.02 \mathrm{~mol} \cdot \mathrm{L}^{-1}$, and sodium hydroxide $0.1 \mathrm{~mol} \cdot \mathrm{L}^{-1}$ ) and $5 \mathrm{~mL}$ of dichloromethane were added one by one to $5 \mathrm{~mL}$ of sample solution. The mixture was vortexed for 1 minute and then centrifuged at $3500 \mathrm{rpm}$ for 15 minutes; following this, the organic layer was collected and spectrophotometrically measured at $365 \mathrm{~nm}$ (UV-1801; Beijing Rayleigh Analytical Instrument Corporation, China). The total amount of $\mathrm{mPEG}_{2000}$-DSPE in the formulation was determined using the same analytical procedure. The amount of $\mathrm{mPEG}_{2000}-\mathrm{DSPE}$ in the sample solution was calculated from the calibration curve of the standard $\mathrm{mPEG}_{2000}-\mathrm{DSPE}$ solution.

\section{Measurement of particle size and zeta potential}

The mean particle size and zeta potential of the SLNs were determined in purified water at $23^{\circ} \mathrm{C}$ by the dynamic light scattering method, using a submicron particle sizer $\left(\right.$ Nicomp $^{\circledR}$ 380; Particle Sizing Systems, Inc, Santa Barbara, CA). The zeta potential was calculated from the electrophoretic mobility, using the Smoluchowski equation.

\section{Biodistribution of the second dose of PEGylated SLNs in mice}

The male Kunming mice were randomly divided into four groups of five: a control group and three treated groups. For the initial injection, the control mice were given 5\% glucose solution and the treated mice were injected with 5, 10, and $20 \mathrm{~mol} \%$ PEG SLNs at a dose of $10 \mu \mathrm{mol}$ phospholipids $\cdot \mathrm{kg}^{-1}$ via the tail vein separately. Seven days later, all the groups received $10 \mathrm{~mol} \%$ PEG SLNs $\left(10 \mu \mathrm{mol}\right.$ phospholipids $\left.\cdot \mathrm{kg}^{-1}\right)$. The detailed injection scheme is presented in Table 2. At specified time points following the second injection, blood was collected, the mice were sacrificed, and samples of liver and spleen were removed. The blood samples were centrifuged at $4000 \mathrm{rpm}$ for 10 minutes to separate the plasma. Plasma and tissue samples were stored at $-20^{\circ} \mathrm{C}$ until analysis.

\section{Pharmacokinetics of the second dose of PEGylated SLNs in beagles}

The beagles were randomly divided into three groups of three, and for the first injection, the beagles were given

Table I Components of each formulation ${ }^{\mathrm{a}}$

\begin{tabular}{llll}
\hline Composition & $\mathbf{5}$ mol\% PEG SLNs & 10 mol\% PEG SLNs & 20 mol\% PEG SLNs \\
\hline GMS (mg) & 100.0 & 100.0 & 100.0 \\
S75 (mg) & 37.1 & 35.2 & 31.2 \\
mPEG ${ }_{2000}$-DSPE (mg) & 7.0 & 14.0 & 28.0 \\
TN (mg) & 20.0 & 20.0 & 20.0 \\
$5 \%$ glucose $(\mathrm{mL})$ & 9.8 & 9.8 & 9.8 \\
\hline
\end{tabular}

Note: aln each formulation, the molar ratio of total phospholipid (S75 and mPEG 2000 -DSPE) was fixed: S75: $\mathrm{mPEG}$ 2000 $-\mathrm{DSPE}=(\mathrm{I}-\mathrm{x}$ ): $x$ (with $x$ being $0.05,0.1$, or 0.2). Abbreviations: GMS, glycerol monostearate; mPEG $_{2000}$-DSPE, N-(carbonyl-methoxypolyethylene-glycol-2000)-I,2-distearoyl-sn-glycero-3-phosphoethanolamine; S75, injectable soybean lecithin; TN, tocopheryl nicotinate; SLN, solid lipid nanoparticle. 
Table 2 Injection scheme of the PEGylated solid lipid nanoparticles (SLNs)

\begin{tabular}{lll}
\hline $\begin{array}{l}\text { Experimental } \\
\text { animal }\end{array}$ & First treatment & Second treatment \\
\hline Mouse & $\begin{array}{l}5 \% \text { glucose solution } \\
5 \mathrm{~mol} \% \text { PEG SLNs }\end{array}$ & $10 \mathrm{~mol} \%$ PEG SLNs \\
& $10 \mathrm{~mol} \%$ PEG SLNs & \\
& $20 \mathrm{~mol} \%$ PEG SLNs & \\
Beagle & $5 \mathrm{~mol} \%$ PEG SLNs & 5 mol\% PEG SLNs \\
& $10 \mathrm{~mol} \%$ PEG SLNs & $10 \mathrm{~mol} \%$ PEG SLNs \\
& 20 mol\% PEG SLNs & $20 \mathrm{~mol} \%$ PEG SLNs \\
\hline
\end{tabular}

Abbreviation: PEG, polyethylene glycol.

SLNs modified with different PEG surface densities at a dose of $2 \mu$ mol phospholipids $\cdot \mathrm{kg}^{-1}$ via a vein in the right forelimb. Blood was collected from the same limb at selected time points using disposable cannulae. Seven days after the first injection, the beagles received a second injection in the same manner as the first injection and blood samples were collected as before. Pharmacokinetic parameters were calculated using DAS software (v 2.0; DAS Software, Inc, Kingston, Ontario, Canada). The detailed injection scheme is presented in Table 2 .

The concentrations of TN in plasma and tissue samples were assayed by HPLC. The analysis system was equipped with a P230 pump and a UV230 ultraviolet and visible spectrophotometer (Dalian Elite Analytical Instruments Co, Ltd, Dalian, Liaoning, China), operated at $264 \mathrm{~nm}$, and a Hypersi ${ }^{\circledR}$ BDS C18 column (Thermo Fisher Scientific, Waltham, MA, $200 \times 4.6 \mathrm{~mm}$ ) filled with particles $5 \mu \mathrm{m}$ in diameter. The mobile phase was methanol/isopropanol $(80 / 20, \mathrm{v} / \mathrm{v})$, the flow rate was $1 \mathrm{~mL} \cdot \mathrm{min}^{-1}$ at $30^{\circ} \mathrm{C}$ and the limit of quantitation was $0.04 \mu \mathrm{g} \cdot \mathrm{mL}^{-1}$. The linearity was excellent over the range of $0.5-100 \mu \mathrm{g} \cdot \mathrm{mL}^{-1}(\mathrm{r}=0.9962, \mathrm{n}=8$ for beagles and $\mathrm{r}=0.9990$, $\mathrm{n}=8$ for mice). It was shown that the extractive recoveries were from $93.45 \%$ to $104.73 \%$ and the precision and accuracy of this HPLC method met the measurement requirements. Samples of plasma and tissue were treated as follows: $100 \mu \mathrm{L}$ of methanol, $100 \mu \mathrm{L}$ of internal standard (tocopheryl acetate), and $600 \mu \mathrm{L}$ of $n$-hexane were added to $100 \mu \mathrm{L}$ of plasma or homogenates (equivalent to $0.1 \mathrm{~g}$ tissue). The mixture was vortexed for 5 minutes and then centrifuged at $10000 \mathrm{rpm}$ for 10 minutes; following this, $500 \mu \mathrm{L}$ of supernatant was collected and dried using a CentriVap ${ }^{\circledR}$ Centrifugal Vacuum Concentrator (Labconco Corporation, Kansas City, MO). Following exsiccation, the dried material was mixed with $100 \mu \mathrm{L}$ of mobile phase, vortexed for 1 minute, and then centrifuged at $10000 \mathrm{rpm}$ for 10 minutes; the supernatant was analyzed using a sample injection volume of $20 \mu \mathrm{L}$.
Detection of anti-PEG immunoglobulin $M$ antibody in beagle serum

It has been proposed that the anti-PEG immunoglobulin M (IgM) produced by the initial dose was responsible for the ABC phenomenon. ${ }^{23,26,30}$ So, to examine the ability of PEGylated SLNs to generate anti-PEG IgM, serum samples were collected 7 days after a single dose of PEGylated SLNs and 24 hours following the second injection, while the serum collected before the first injection was used as a control. Anti-PEG IgM was then determined according to a slightly modified previously published procedure. ${ }^{24}$ Briefly, $10 \mathrm{nmol}$ $\mathrm{mPEG}_{2000}$-DSPE in $50 \mu \mathrm{L}$ of $100 \%$ ethanol was added to each well of a 96-well microplate (Corning Incorporated, New York, NY) and thoroughly air-dried. Wells were then blocked with $100 \mu \mathrm{L}$ of $1 \%$ bovine serum albumin (Biosharp, Korea) in $50 \mathrm{mM}$ Tris-buffered saline (pH 8.0) for 30 minutes. The wells were then washed five times with Tris-buffered saline (Sigma-Aldrich, St Louis, MO) containing $0.05 \%$ Tween ${ }^{\circledR} 20$ (Sigma-Aldrich, St Louis, MO). Diluted serum samples (1:200 dilution in 1\% bovine serum albumin/0.05\% Tween 20/Tris-buffered saline) (100 $\mu \mathrm{L}$ ) were then applied to the wells, followed by incubation for 30 minutes and washing five times, as previously described. Horseradish peroxidase-conjugated goat anti-dog IgM antibody $\left(100 \mu \mathrm{L}, 1 \mu \mathrm{g} \cdot \mathrm{mL}^{-1}\right.$, goat anti-dog IgM was purchased from Immunology Consultants Laboratory, Inc, Oregon, USA, then the antibody was conjugated with HRP by Beijing Biosynthesis Biotechnology Co, Ltd, Beijing, China) was added to each well. After a 30-minute incubation, the wells were washed five times, as previously described. Staining was carried out by the addition of $100 \mu \mathrm{L}$ of $o$-phenylenediamine $\left(1 \mathrm{mg} \cdot \mathrm{mL}^{-1}\right)$ from Sigma-Aldrich. After a 5-minute incubation period, the reaction was stopped by adding $100 \mu \mathrm{L}$ of $2 \mathrm{~N}$ sulfuric acid, and the absorbance was measured at $490 \mathrm{~nm}$ using a microplate reader (Bio-Rad Laboratories Ltd, Hemel Hempstead, Hertfordshire, United Kingdom). All incubations were performed at $25^{\circ} \mathrm{C}$.

\section{Statistical analysis}

All mean values are expressed as mean plus or minus standard deviation. Statistical analysis was performed by Student's $t$-test using SPSS software (SPSS, Inc, Chicago, IL). A $P$-value $<0.05$ was considered statistically significant.

\section{Results}

\section{The characteristics of PEGylated SLNs}

It has been reported that the in vivo characteristics of nanocarriers may be influenced by many factors 
including composition, particle size, and zeta potential. ${ }^{33,34}$ Therefore, the SLNs used for the present study needed to be fully characterized. The authors prepared three kinds of SLNs - containing 5, 10, and $20 \mathrm{~mol}^{\%} \mathrm{mPEG}_{2000}$-DSPE, respectively. The mean particle size of the prepared SLNs was $120-130 \mathrm{~nm}$, with a zeta potential of approximately $-20 \mathrm{mV}$. All the SLN formulations were sterilized by autoclaving at $100^{\circ} \mathrm{C}$ at 0.45 bar for 30 minutes, and the SLNs were physically stable during sterilization. Details with regard to the pharmaceutical properties of the prepared SLNs are summarized in Table 3.

\section{The incorporated ratio of $\mathrm{mPEG}_{2000}-\mathrm{DSPE}$ in SLNs}

The PEG surface density is reported to play an important role in inducing the $\mathrm{ABC}$ phenomenon. The present authors determined the incorporated ratio of $\mathrm{MPEG}_{2000}$-DSPE in SLNs by the picrate method. The calibration curve for the standard mPEG $_{2000}$-DSPE solution was $A=0.00861 C+0.0971$ in the range of $3-36 \mu \mathrm{g} \cdot \mathrm{mL}^{-1}(\mathrm{r}=0.9935 ; \mathrm{n}=8$; where $A$ is absorbance and $C$ is $\mathrm{mPEG}_{2000}$-DSPE concentration), and the incorporated ratios of 5,10, and $20 \mathrm{~mol} \%$ PEG SLNs were $90.21 \% \pm 7.20 \%, 92.93 \% \pm 5.44 \%$, and $89.37 \% \pm 4.01 \%$, respectively. These results indicate that almost all of the $\mathrm{mPEG}_{2000}$-DSPE was incorporated into the SLNs.

\section{Biodistribution of the second dose of PEGylated SLNs in mice}

At present, most $\mathrm{ABC}$ studies are performed in mice or rats; therefore, the authors initially investigated the $\mathrm{ABC}$ of PEGylated SLNs in mice.

As shown in Figure 1, PEGylated SLNs injected weekly dramatically influenced the blood clearance in mice. SLNs containing $10 \mathrm{~mol} \% \mathrm{PEG}$ produced a higher elimination rate and more hepatic and splenic uptake of the second dose than with the other two formulations (Figure 2). For $10 \mathrm{~mol} \%$ PEG SLNs, the biodistribution 30 minutes after the second dose showed a significantly reduced plasma concentration (from $71.30 \% \pm 9.36 \%$ to $42.56 \% \pm 9.69 \%$ of the injected dose) and, concomitantly, a higher accumulation in the liver (from $5.35 \% \pm 0.43 \%$ to $23.29 \% \pm 2.78 \%$ of the injected dose) (Figure 2A). Significant accumulation of the second dose was also observed in the spleen (Figure 2B), although it was substantially lower than in the liver. These results clearly indicate that the PEGylated SLNs were able to induce the $\mathrm{ABC}$ phenomenon.

\section{Effect of circulation time on the $A B C$ phenomenon induced in beagles}

Reports have shown that the PEG surface density affects the induction of the ABC phenomenon. ${ }^{29}$ Furthermore, the present authors' results from mice also indicate that the amount of PEG lipid presented in the SLNs plays an important role in the induction of the $\mathrm{ABC}$ phenomenon. The authors speculated that varying circulation times of the initial dose were a result of different PEG surface densities and that this was the underlying factor in the induction of the different ABC phenomena. Thus, to further investigate whether a long circulation time is required to induce the enhanced clearance effect, the authors selected the beagle as the experimental animal - it was helpful to study the pharmacokinetics of the two doses in the same animal.

The beagles were first treated with SLNs containing 5,10 , and $20 \mathrm{~mol}^{2} \mathrm{mPEG}_{2000}$-DSPE, at a dose of $2 \mu \mathrm{mol}$ phospholipids $\cdot \mathrm{kg}^{-1}$, and the same dose was also administered 7 days later. As shown in Table 4, PEGylated SLNs exhibited a gradually increased circulation time with increasing PEG surface density; Figure 3 shows that all kinds of PEGylated SLNs induced the ABC phenomenon in beagles.

Conventionally, subsequent doses are identical to allow for investigation of the influence of the first injection.

Table 3 Physicochemical parameters of solid lipid nanoparticles (SLNs) before and after sterilization

\begin{tabular}{lccc}
\hline Physicochemical parameter & $\mathbf{5 ~ m o l \% ~ P E G ~ S L N s ~}$ & 10 mol\% PEG SLNs & 20 mol\% PEG SLNs \\
\hline Average diameter $(\mathrm{nm})$ & & & $130.5 \pm 7.8$ \\
$\quad$ Before & $138.0 \pm 2.9$ & $131.5 \pm 4.1$ & $119.3 \pm 5.5$ \\
$\quad$ After & $132.5 \pm 3.8$ & $127.5 \pm 3.9$ & $-16.98 \pm 1.9$ \\
Zeta potential $(\mathrm{mV})$ & & & $-17.66 \pm 3.5$ \\
$\quad$ Before & $-27.13 \pm 3.0$ & $-21.78 \pm 2.4$ & \\
$\quad$ After & $-28.52 \pm 2.4$ & $-22.65 \pm 3.8$ & $97.21 \pm 1.38$ \\
EE\% & $95.32 \pm 2.58$ & $94.42 \pm 1.09$ & $93.21 \pm 2.01$ \\
$\quad$ Before & $90.97 \pm 4.82$ & $91.32 \pm 3.21$ & \\
After &
\end{tabular}

Abbreviations: EE\%, encapsulation efficiency; PEG, polyethylene glycol. 


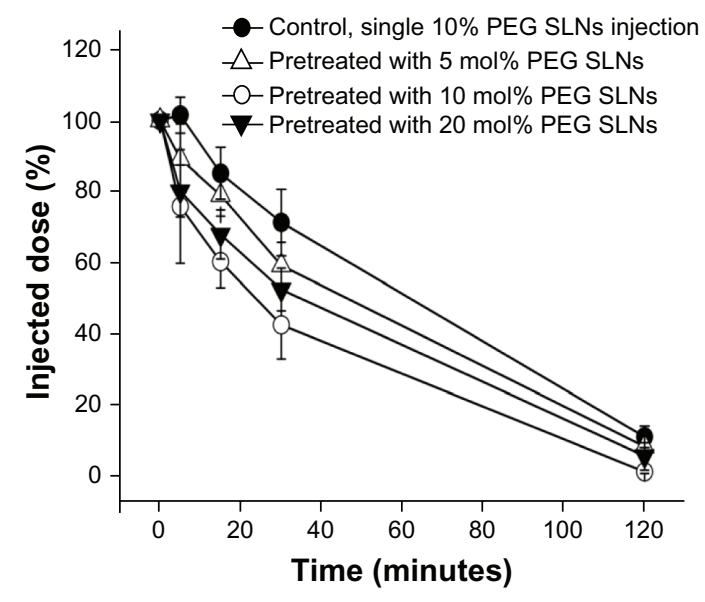

Figure I Effect of the prior injection of solid lipid nanoparticles (SLNs) modified with different polyethylene glycol (PEG) surface-densities on the pharmacokinetics of a second dose of 10 mol\% PEG SLNs in mice. The mice were pre-administered SLNs containing 5, 10, and 20 mol\% mPEG ${ }_{2000}$-DSPE [N-(carbonyl-methoxypolyethyleneglycol-2000)-1,2-distearoyl-sn-glycero-3-phosphoethanolamine] at a dose of $10 \mu \mathrm{mol}$ phospholipids $\cdot \mathrm{kg}^{-1}$. Seven days later, 10 mol\% PEG SLNs were injected intravenously at the same dose. Mice pretreated with an injection of glucose served as controls. Notes: Data are shown as mean plus or minus standard deviation; mice were randomly divided into four groups of five.

However, the subsequent dose, as the effectuation phase in the ABC phenomenon, ${ }^{6}$ was independent of this effect. Therefore, as long as it can determine the extent of the induced $\mathrm{ABC}$ phenomenon, the influence of the first dose is better studied even if subsequent doses are different. In this study, the ratio of $\mathrm{AUC}_{(0-t)}$ for the second injection to that of the first injection was selected as the $\mathrm{ABC}$ index, used to evaluate the phenomenon, and a higher index meant a weaker induction of the $\mathrm{ABC}$ phenomenon. ${ }^{8}$ The authors calculated the ratio for the different time intervals, which showed that the $\mathrm{ABC}$ index exhibited significant difference within 30 minutes following the second injection (Table 4), and the discrepancies in the ratio basically remained unchanged until 24 hours after the second injection (data not shown). These results imply that the $\mathrm{ABC}$ effect lasted only a short time, and the ratio of $\mathrm{AUC}_{(0-30 \mathrm{~min})}$ (ie, area under the concentration-time curve from time 0 to 30 minutes) was suitable for evaluating this phenomenon. Also, the ABC level induced by SLNs was, in descending order. 10, 20, and 5 mol\% PEG SLNs, and $10 \mathrm{~mol} \%$ PEG SLNs induced the most marked ABC phenomenon in beagles, which is consistent with the results obtained in mice.

The physical state of the beagles was not influenced by the quantities of blood collected. In addition, the single treatment with PEGylated SLNs did not produce any visible anaphylactic reaction. However, upon subsequent administration, noticeable hypersensitive reactions were observed in the beagles. Symptoms developed within
A

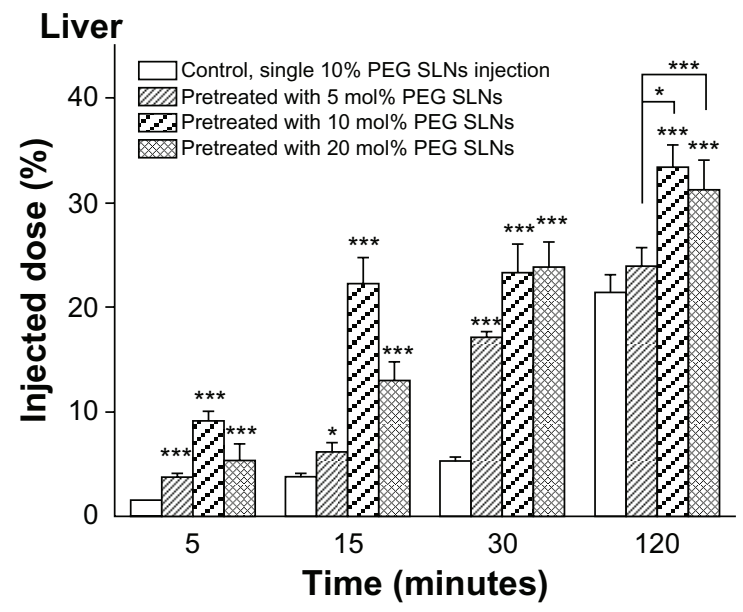

B Spleen

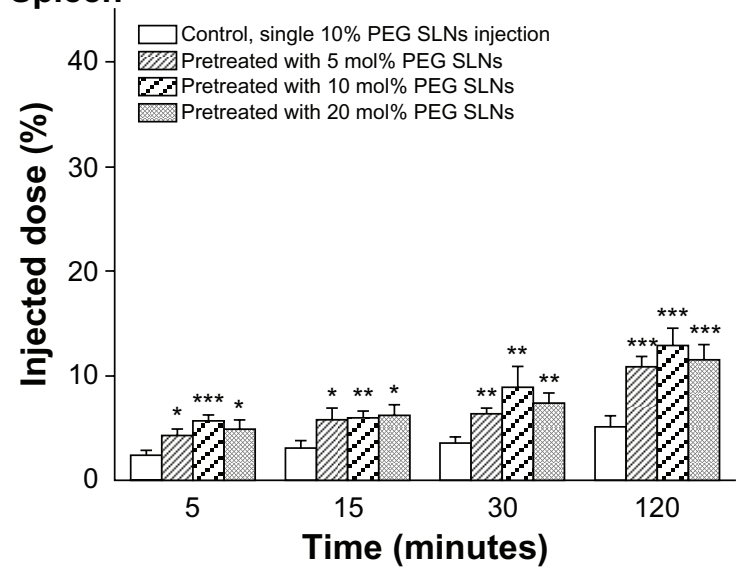

Figure 2 Hepatic (A) and splenic (B) accumulation of the PEGylated solid lipid nanoparticles (SLNs) in mice at different time intervals after the second injection. The mice were pre-administered SLNs containing 5, 10, and $20 \mathrm{~mol}^{2} \mathrm{mPEG}_{2000}$-DSPE [N-(carbonyl-methoxypolyethylene-glycol-2000)- I,2-distearoyl-sn-glycero-3phosphoethanolamine] at a dose of $10 \mu \mathrm{mol}$ phospholipids $\cdot \mathrm{kg}^{-1}$. Seven days later, $10 \mathrm{~mol} \%$ polyethylene glycol (PEG) SLNs were intravenously injected at the same dose. Mice pretreated with an injection of glucose served as controls.

Notes: Data are shown as mean plus or minus standard deviation; $P$-values apply to difference between control and treated mice; $* P<0.05$; $* * P<0.01$; $* * * P<0.00$ I; mice were randomly divided into four groups of five.

30 minutes after readministration and these included lethargy, licking of lips, lachrymation, labored respiration, and nausea, which are typical of an Ab-mediated anaphylactic reaction. ${ }^{35}$ Establishment of hypersensitivity might suggest the $\mathrm{ABC}$ phenomenon was induced to a certain degree, while it seemed there was no direct relationship between the duration of the symptoms and the intensity of this phenomenon. The authors also measured the serum levels of IgM antibodies reactive to PEG in the beagles after injection of PEGylated SLNs. The results indicate that after an initial priming dose of PEGylated SLNs, each of the formulations led to production of anti-PEG IgM to different degrees. At 24 hours following the second injection, the production returned almost to its original level or even lower, suggesting that anti-PEG IgM was responsible for the $\mathrm{ABC}$ phenomenon (Figure 4). However, there was not 
Table 4 The main pharmacokinetic parameters of the injected PEGylated solid lipid nanoparticles (SLNs) in beagles

\begin{tabular}{|c|c|c|c|c|c|}
\hline \multirow[t]{2}{*}{ Injected dose } & \multirow{2}{*}{$\operatorname{AUC}_{(0-24 \mathrm{~h})}(\mathrm{mg} / \mathrm{L} * \mathrm{~h})$} & \multirow[t]{2}{*}{ CL (L/h/kg) } & \multirow[t]{2}{*}{$T_{1 / 2 \beta}(h)$} & \multicolumn{2}{|l|}{$A B C$ index ${ }^{a}$} \\
\hline & & & & AUC $_{(0-30 \mathrm{~min})}$ & $\operatorname{AUC}_{(0-24 \mathrm{~h})}$ \\
\hline \multicolumn{6}{|c|}{$5 \mathrm{~mol} \%$ PEG SLNs } \\
\hline First dose & $15.254 \pm 1.167$ & $0.050 \pm 0.008$ & $1.292 \pm 0.123$ & $0.802 \pm 0.072^{* *}$ & $0.752 \pm 0.050^{* * *}$ \\
\hline Second dose & $11.632 \pm 0.942$ & $0.066 \pm 0.005$ & $1.126 \pm 0.092$ & & \\
\hline \multicolumn{6}{|c|}{10 mol\% PEG SLNs } \\
\hline First dose & $90.554 \pm 8.512$ & $0.010 \pm 0.011$ & $3.411 \pm 0.379$ & $0.490 \pm 0.040^{\ldots}$ & $0.376 \pm 0.025^{\ldots}$ \\
\hline Second dose & $34.043 \pm 3.498$ & $0.025 \pm 0.019$ & $1.604 \pm 0.026$ & & \\
\hline \multicolumn{6}{|c|}{$20 \mathrm{~mol} \%$ PEG SLNs } \\
\hline First dose & $136.940 \pm 1 \mid .233$ & $0.006 \pm 0.001$ & $4.192 \pm 0.278$ & $0.600 \pm 0.094^{*, \#}$ & $0.489 \pm 0.037 * * * \ldots$ \\
\hline Second dose & $67.097 \pm 8.940$ & $0.012 \pm 0.007$ & $3.395 \pm 0.237$ & & \\
\hline
\end{tabular}

Notes: ${ }^{a}$ The $A B C$ index was calculated as $A \cup C_{(0-t)}$ of the second dose/AUC $(0-t)$ of the first dose; $* P<0.05 ; * * P<0.01$; $* * * P<0.001$ represent a significant difference compared with 10 mol\% PEG SLNs; ${ }^{P}<0.05$; $P<0.01$; $P<0.001$ represent a significant difference compared with 5 mol\% PEG SLNs; the beagles were randomly divided into three groups of three.

Abbreviations: $A B C$, accelerated blood clearance; $\mathrm{AUC}_{(0-24 \mathrm{~h})}$, area under the concentration-time curve from time 0 to 24 hours; $A U C_{(0-30 \text { min) }}$, area under the concentration-time curve from time 0 to 30 minutes; $A \cup C_{(0-t)}$, area under the concentration-time curve from time 0 to the last measured concentration; $C L$, total body clearance; $P E G$, polyethylene glycol; $T_{1 / 2 \beta}$, mean elimination half-life.

a complete correlation between the level of anti-PEG IgM and the extent of the $\mathrm{ABC}$ phenomenon induced: the level of IgM generated by $10 \mathrm{~mol} \%$ PEG SLNs was not higher than that generated by $20 \mathrm{~mol} \%$ PEG SLNs.

\section{Discussion}

The authors studied the $\mathrm{ABC}$ phenomenon induced by PEGylated SLNs and, in this study, selected the beagle as an experimental animal for the first time. The PEGylated SLNs were found to trigger this phenomenon in both mice and beagles upon repeated injection, with different intensities of the $\mathrm{ABC}$ phenomenon being observed between the two different animal species. The $\mathrm{ABC}$ phenomenon was mostly an immune response and so the nanocarriers were eliminated rapidly when this unexpected phenomenon was induced, owing to the fact that the immune response was elicited immediately when the pathogen and antibody came into contact. Because of this, blood samples were required to be densely collected in a short period of time after the second injection. The beagle has a high blood volume, which protects their physical function when collecting many blood samples over a short time period - more so than other experimental animals such as mice, rats, and rabbits. Furthermore, the beagle is an excellent model when monitoring of hypersensitive reactions is required during the experimental process; again, this is often difficult in other experimental animals such as mice, rats, and rabbits. Therefore, the beagle is an excellent model for examining the induction of the $\mathrm{ABC}$ phenomenon.

The prolonged circulation time in the blood is the most important characteristic of the PEGylated nanocarriers, and the PEG surface density is thought to be the key factor determining this extended circulation time. ${ }^{36}$ However, despite extensive studies on the $\mathrm{ABC}$ phenomenon, it remains unclear whether the circulation time affects the induction of this phenomenon. Due to the $\mathrm{ABC}$ effect lasting only a short time, the $\mathrm{AUC}_{(0-30 \mathrm{~min})}$ ratio was used in the present study as an $\mathrm{ABC}$ index to evaluate the extent of the phenomenon, and the results demonstrated that a marked $\mathrm{ABC}$ phenomenon was induced only when the PEGylated SLNs exhibited a more prolonged circulation time (Table 4). Previous studies have reported that PEGylated nanocarriers might elicit an anti-PEG IgM response in a T-cell-independent manner and appear to be T-cell-independent type 2 antigens ${ }^{24,25}$ - the surfacemodified PEG might act as an antigenic epitope. For most T-cell-independent type 2 antigens, the prolonged contact of the antigens with membrane immunoglobulin and persistent B-cell signaling is common in inducing a humoral immune response in the absence of helper T cells..$^{37,38}$ Consequently, in addition to the density of PEG, it is rational to speculate that extended circulation time also plays an important role in the induction of the ABC phenomenon. Regarding 5 mol\% PEG SLNs, because of the only slightly prolonged circulation time, inefficient contact of the PEGylated SLNs with membrane immunoglobulin stimulated B-cells and resulted in secretion of a small amount of anti-PEG IgM, with only a weaker immune response and insignificant $\mathrm{ABC}$ phenomenon. The $20 \mathrm{~mol} \%$ PEG SLNs exhibited an even more prolonged circulation time, and should have resulted in producing a larger quantity of anti-PEG IgM, while the results show that the $20 \mathrm{~mol} \%$ PEG SLNs and the $10 \mathrm{~mol} \%$ PEG SLNs induced subequal amounts of anti-PEG IgM. After PEGylated SLNs exhibited a clearly prolonged half-life, the authors speculated that the circulation time was no longer a determinant in this phenomenon, and the 

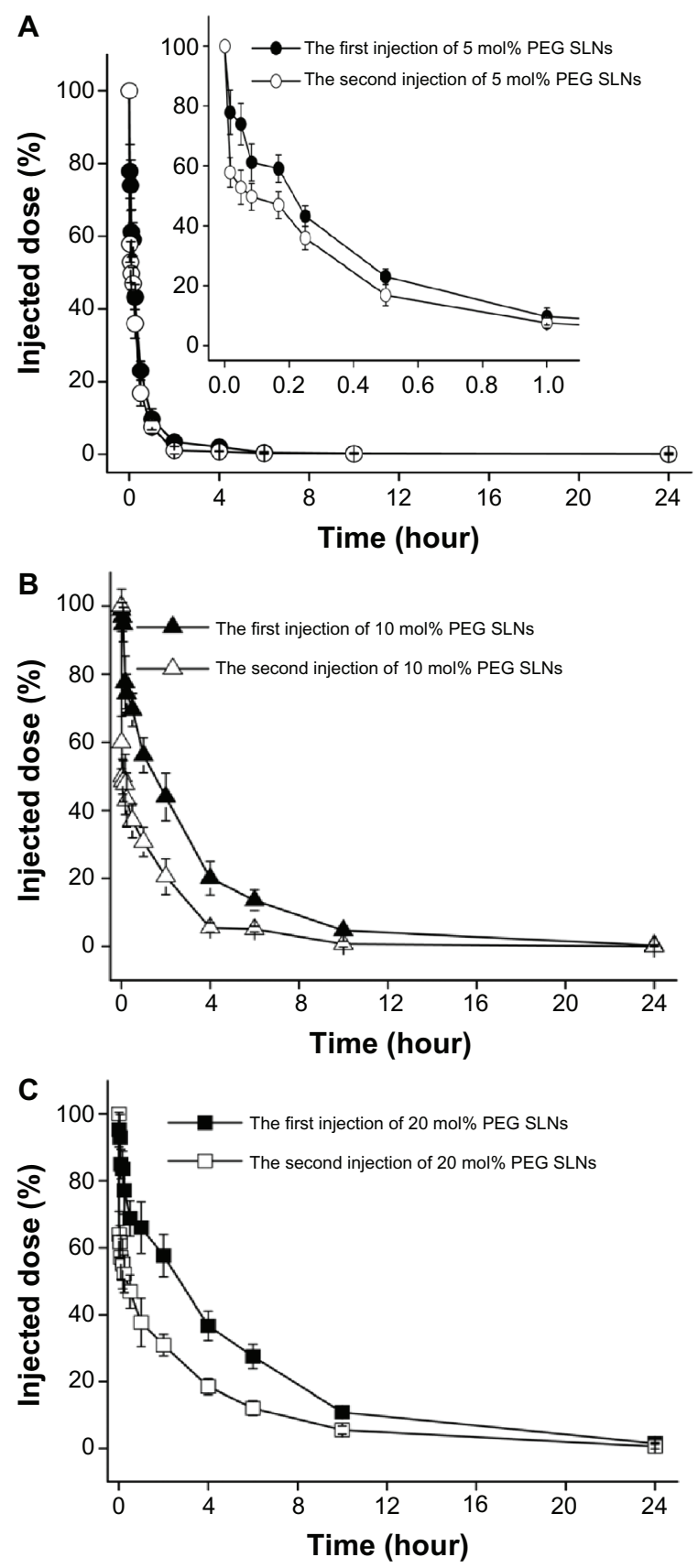

Figure 3 Effect of mPEG 2000 -DSPE [N-(carbonyl-methoxypolyethylene-glycol-2000)- I, 2-distearoyl-sn-glycero-3-phosphoethanolamine] content in solid lipid nanoparticles (SLNs) on the induction of accelerated blood clearance phenomenon in beagles. The beagles were first administered SLNs containing (A) 5, (B) 10, and (C) $20 \mathrm{~mol} \%$ $\mathrm{mPEG}_{2000}-\mathrm{DSPE}$, at a dose of $2 \mu \mathrm{mol}$ phospholipids $\cdot \mathrm{kg}^{-1}$. Seven days after the first injection, the beagles were given the second injection at the same dose.

Notes: Data are shown as mean plus or minus standard deviation; the beagles were randomly divided into three groups of three.

Abbreviation: PEG, polyethylene glycol.

PEG surface density, referred to as the density of the antigenic epitope in the $\mathrm{ABC}$ phenomenon, began to play a decisive role in inducing this immune response. Excessive cross-linking of membrane immunoglobulin induced by the higher surface density of PEG resulted in the receptor rearrangement and

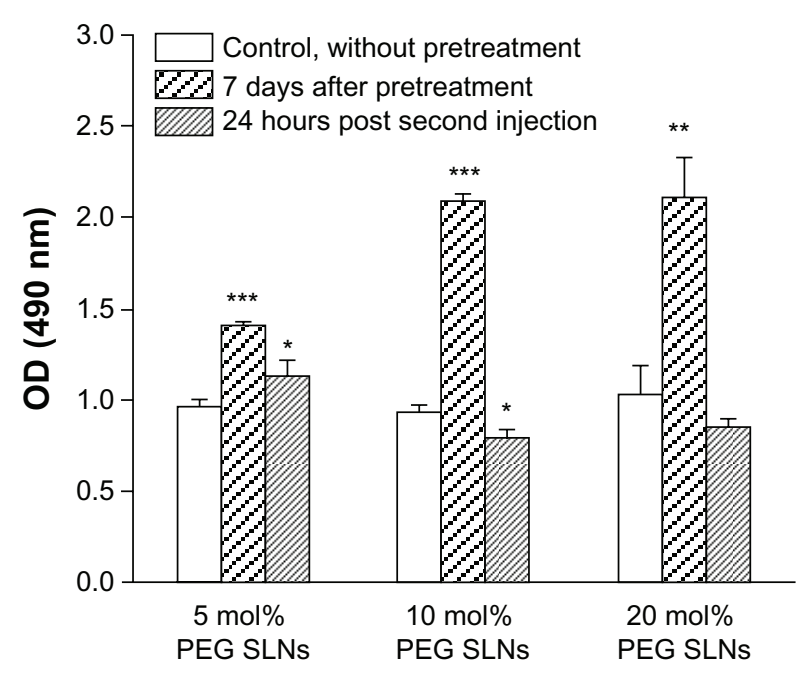

Figure 4 Anti-polyethylene glycol (PEG) immunoglobulin M responses following a single intravenous injection of PEGylated solid lipid nanoparticles (SLNs) in beagles. The beagles were administrated with SLNs containing 5, 10, and $20 \mathrm{~mol}^{2} \mathrm{mPEG}_{2000^{-}}$ DSPE [N-(carbonyl-methoxypolyethylene-glycol-2000)-1,2-distearoyl-sn-glycero-3phosphoethanolamine]. Seven days later, blood was withdrawn and serum was collected. The serum was also collected at 24 hours after a second injection.

Notes: The amount of anti-PEG immunoglobulin $M$ antibody was determined as described in the "Material and methods" section; each value represents the mean plus or minus standard deviation; $P$-values apply to differences against the control (naïve serum); $* P<0.05$; $* * P<0.01$; $* * * P<0.00$ I; the beagles were randomly divided into three groups of three.

Abbreviation: $O D$, optical density.

membrane reordering, ${ }^{39}$ leading to the $20 \mathrm{~mol} \%$ PEG SLNs being able to induce an ineffective immune response and a relatively attenuated $\mathrm{ABC}$, consistent with other reports in the literature..$^{29}$

In this study, the authors initially proposed that a relatively extended circulation time of the initial dose of PEGylated SLNs might be the major underlying factor determining induction of the $\mathrm{ABC}$ phenomenon. The $10 \mathrm{~mol} \%$ PEG SLNs mostly exhibited a suitable circulation time in this study and they effectively stimulated B cells; thus, the activated B cells secreted a great deal of anti-PEG IgM, consequently binding IgM to the sequentially injected PEGylated SLNs and resulting in the $10 \mathrm{~mol} \%$ PEG SLNs producing a marked $\mathrm{ABC}$ effect. While a study by Laverman et $\mathrm{al}^{6}$ showed that the circulation time of liposomes did not influence the induction of $\mathrm{ABC}$, it should be noted that conventional liposomes of different particle sizes were employed to evaluate the effect of the circulation time in the Laverman et $\mathrm{al}^{6}$ study, while it is not known in the present study whether conventional nanocarriers and PEGylated nanocarriers exhibited the same mechanism for this phenomenon.

\section{Conclusion}

The PEGylated SLNs were found to induce the ABC phenomenon upon repeated injection, both in mice 
and in beagles. In addition, the authors found the beagle to be the most suitable model for examination of the ABC phenomenon. Another major factor determining the induction of the $\mathrm{ABC}$ phenomenon may be the circulation time of PEGylated SLNs, although further investigation of a defined range of circulation times over which the $\mathrm{ABC}$ phenomenon would not be induced is needed. These findings may provide new perspectives in our understanding of the mechanism of the $\mathrm{ABC}$ phenomenon.

\section{Acknowledgment}

The National Natural Science Foundation of China supported this study (Grant No 81072602).

\section{Disclosure}

The authors report no conflicts of interest in this work.

\section{References}

1. Nie S. Understanding and overcoming major barriers in cancer nanomedicine. Nanomedicine (Lond). 2010;5(4):523-528.

2. Storm G, Belliot SO, Daemen T, Lasic DD. Surface modification of nanoparticles to oppose uptake by the mononuclear phagocyte system. Adv Drug Deliv Rev. 1995;17(1):31-48.

3. Allen TM, Hansen C, Martin F, Redemann C, Yau-Young A. Liposomes containing synthetic lipid derivatives of poly(ethylene glycol) show prolonged circulation half-lives in vivo. Biochim Biophys Acta. 1991; 1066(1):29-36.

4. Woodle MC, Lasic DD. Sterically stabilized liposomes. Biochim Biophys Acta. 1992;1113(2):171-199.

5. Stolnik S, Illum L, Davis SS. Long circulating microparticulate drug carriers. Adv Drug Deliv Rev. 1995;16(2-3):195-214.

6. Laverman P, Carstens MG, Boerman OC, et al. Factors affecting the accelerated blood clearance of polyethylene glycol-liposomes upon repeated injection. J Pharmacol Exp Ther. 2001;298(2):607-612.

7. Dams ET, Laverman P, Oyen WJ, et al. Accelerated blood clearance and altered biodistribution of repeated injections of sterically stabilized liposomes. J Pharmacol Exp Ther. 2000;292(3):1071-1079.

8. Ishihara T, Takeda M, Sakamoto H, et al. Accelerated blood clearance phenomenon upon repeated injection of PEG-modified PLA-nanoparticles. Pharm Res. 2009;26(10):2270-2279.

9. Lu W, Wan J, She Z, Jiang X. Brain delivery property and accelerated blood clearance of cationic albumin conjugated PEGylated nanoparticle. J Control Release. 2007;118(1):38-53.

10. Koide H, Asai T, Hatanaka K, et al. Particle size-dependent triggering of accelerated blood clearance phenomenon. Int J Pharm. 2008;362(1-2): 197-200.

11. Tagami T, Nakamura K, Shimizu T, Ishida T, Kiwada H. Effect of siRNA in PEG-coated siRNA-lipoplex on anti-PEG IgM production. J Control Release. 2009;137(3):234-240.

12. Tagami T, Uehara Y, Moriyoshi N, Ishida T, Kiwada H. Anti-PEG IgM production by siRNA encapsulated in a PEGylated lipid nanocarrier is dependent on the sequence of the siRNA. J Control Release. 2011; 151(2):149-154.

13. Kaminskas LM, McLeod VM, Porter CJ, Boyd BJ. Differences in colloidal structure of PEGylated nanomaterials dictate the likelihood of accelerated blood clearance. J Pharm Sci. 2011;100(11):5069-5077.

14. Müller RH, Mäder K, Gohla S. Solid lipid nanoparticles (SLN) for controlled drug delivery: a review of the state of the art. Eur J Pharm Biopharm. 2000;50(1):161-177.
15. Wong HL, Bendayan R, Rauth AM, Li Y, Wu XY. Chemotherapy with anticancer drugs encapsulated in solid lipid nanoparticles. Adv Drug Deliv Rev. 2007:59(6):491-504.

16. Yang SC, Lu LF, Cai Y, Zhu JB, Liang BW, Yang CZ. Body distribution in mice of intravenously injected camptothecin solid lipid nanoparticles and targeting effect on brain. J Control Release. 1999;59(3): 299-307.

17. Parveen S, Misra R, Sahoo SK. Nanoparticles: a boon to drug delivery, therapeutics, diagnostics and imaging. Nanomedicine. 2012;8(2): $147-166$.

18. Wong HL, Bendayan R, Rauth AM, Wu XY. Development of solid lipid nanoparticles containing ionically complexed chemotherapeutic drugs and chemosensitizers. J Pharm Sci. 2004;93(8):1993-2008.

19. Kang KW, Chun MK, Kim O, et al. Doxorubicin-loaded solid lipid nanoparticles to overcome multidrug resistance in cancer therapy. Nanomedicine. 2010;6(2):210-213.

20. Wong HL, Bendayan R, Rauth AM, Xue HY, Babakhanian K, Wu XY. A mechanistic study of enhanced doxorubicin uptake and retention in multidrug resistant breast cancer cells using a polymer-lipid hybrid nanoparticle system. J Pharmacol Exp Ther. 2006;317(3):1372-1381.

21. Zhang XG, Miao J, Dai YQ, Du YZ, Yuan H, Hu FQ. Reversal activity of nanostructured lipid carriers loading cytotoxic drug in multi-drug resistant cancer cells. Int J Pharm. 2008;361(1-2):239-244.

22. Wong HL, Rauth AM, Bendayan R, et al. A new polymer-lipid hybrid nanoparticle system increases cytotoxicity of doxorubicin against multidrug-resistant human breast cancer cells. Pharm Res. 2006;23(7): 1574-1585.

23. Ishida T, Ichihara M, Wang X, et al. Injection of PEGylated liposomes in rats elicits PEG-specific IgM, which is responsible for rapid elimination of a second dose of PEGylated liposomes. J Control Release. 2006; 112(1):15-25.

24. Ishida T, Wang X, Shimizu T, Nawata K, Kiwada H. PEGylated liposomes elicit an anti-PEG IgM response in a T cell-independent manner. J Control Release. 2007;122(3):349-355.

25. Koide H, Asai T, Hatanaka K, et al. T cell-independent B cell response is responsible for $\mathrm{ABC}$ phenomenon induced by repeated injection of PEGylated liposomes. Int J Pharm. 2010;392(1-2):218-223.

26. Wang X, Ishida T, Kiwada H. Anti-PEG IgM elicited by injection of liposomes is involved in the enhanced blood clearance of a subsequent dose of PEGylated liposomes. J Control Release. 2007;119(2):236-244.

27. Ishida T, Ichikawa T, Ichihara M, Sadzuka Y, Kiwada H. Effect of the physicochemical properties of initially injected liposomes on the clearance of subsequently injected PEGylated liposomes in mice. J Control Release. 2004;95(3):403-412.

28. Ishida T, Atobe K, Wang X, Kiwada H. Accelerated blood clearance of PEGylated liposomes upon repeated injections: effect of doxorubicinencapsulation and high-dose first injection. J Control Release. 2006; 115(3):251-258

29. Ishida T, Harada M, Wang XY, Ichihara M, Irimura K, Kiwada H. Accelerated blood clearance of PEGylated liposomes following preceding liposome injection: effects of lipid dose and PEG surfacedensity and chain length of the first-dose liposomes. J Control Release. 2005;105(3):305-317.

30. Sroda K, Rydlewski J, Langner M, Kozubek A, Grzybek M, Sikorski AF. Repeated injections of PEG-PE liposomes generate anti-PEG antibodies. Cell Mol Biol Lett. 2005;10(1):37-47.

31. Shimada K, Matsuo S, Sadzuka Y, et al. Determination of incorporated amounts of poly(ethylene glycol)-derivatized lipids in liposomes for the physicochemical characterization of stealth liposomes. Int J Pharm. 2000;203(1-2):255-263.

32. Sadzuka Y, Nakade A, Tsuruda T, Sonobe T. Study on the characterization of mixed polyethyleneglycol modified liposomes containing doxorubicin. J Control Release. 2003;91(3):271-280.

33. Aggarwal P, Hall JB, McLeland CB, Dobrovolskaia MA, McNeil SE. Nanoparticle interaction with plasma proteins as it relates to particle biodistribution, biocompatibility and therapeutic efficacy. Adv Drug Deliv Rev. 2009;61(6):428-437. 
34. Alexis F, Pridgen E, Molnar LK, Farokhzad OC. Factors affecting the clearance and biodistribution of polymeric nanoparticles. Mol Pharm. 2008;5(4):505-515.

35. Roy K, Mao HQ, Huang SK, Leong KW. Oral gene delivery with chitosan: DNA nanoparticles generates immunologic protection in a murine model of peanut allergy. Nat Med. 1999;5(4):387-391.

36. Dos Santos N, Allen C, Doppen AM, et al. Influence of poly(ethylene glycol) grafting density and polymer length on liposomes: relating plasma circulation lifetimes to protein binding. Biochim Biophys Acta. 2007; 1768(6):1367-1377.
37. Humphrey JH. Tolerogenic or immunogenic activity of haptenconjugated polysaccharides correlated with cellular localization. Eur J Immunol. 1981;11(3):212-220.

38. Mond JJ, Vos Q, Lees A, Snapper CM. T cell independent antigens. Curr Opin Immunol. 1995;7(3):349-354.

39. Basten A, Silveira PA. B-cell tolerance: mechanisms and implications. Curr Opin Immunol. 2010;22(5):566-574.

\section{Publish your work in this journal}

The International Journal of Nanomedicine is an international, peerreviewed journal focusing on the application of nanotechnology in diagnostics, therapeutics, and drug delivery systems throughout the biomedical field. This journal is indexed on PubMed Central, MedLine, CAS, SciSearch $\AA$, Current Contents ${ }^{\circledR} /$ Clinical Medicine,
Journal Citation Reports/Science Edition, EMBase, Scopus and the Elsevier Bibliographic databases. The manuscript management system is completely online and includes a very quick and fair peer-review system, which is all easy to use. Visit http://www.dovepress.com/ testimonials.php to read real quotes from published authors. 